EPiC Series in Computing
Volume 75, 2021, Pages 11-21
CAINE 2020. The 33rd International Conference on
Computer Applications in Industry and Engineering

\title{
Digital Transformation: A Quality Model Based on ISO 25010 and User Experience
}

\author{
Narayan Debnath ${ }^{1}$, Mario Peralta ${ }^{2}$, Carlos Salgado ${ }^{2}$, Lorena Baigorria ${ }^{2}$, \\ Daniel Riesco ${ }^{2}$, Germán Montejano ${ }^{2}$, Mouna Mazzi ${ }^{3}$ \\ ${ }^{1}$ School of Computing and Information Technology \\ Eastern International University, Vietnam \\ narayan.debnath@eiu.edu.vn \\ ${ }^{2}$ Departamento de Informática Facultad de Ciencias Físico-Matemáticas y \\ Naturales Universidad Nacional de San Luis, Ejército de los Andes 950. San Luis, C.P. 5700, \\ \{mperalta, csalgado, flbaigor, driesco, gmonte\}@unsl.edu.ar \\ ${ }^{3}$ University Mohammed V, Avenue des Nations Unies, Rabat, 10000. \\ mazzi.mouna@gmail.com
}

\begin{abstract}
To think about the experience that the user will have when interacting with a piece of software, is to begin to understand that, in the process of interaction, different factors influence: individual, social, contextual, cultural and those of the product in question. So, there is a need to have some strategy, technique, model or method that allows to systematically organize the tasks to help software developers and professionals from different disciplines to work together for the development of software interfaces. The objective of this paper is to provide software development companies with a quality model that serves as an instrument, guide or good practice that allows them to position themselves at a highly competitive level in the current market through production of quality software based on user experience. A quality model is proposed for which the structure of the International Standard ISO 13407:1999, the ISO 25000 standard and information collected from the software service industry of the region were taken into consideration.
\end{abstract}

\section{Introduction}

While starting a software development process, it is reasonable to analyze how to improve the user experience through efficient utilization of the available resources. To better understand this issue, we encounter the term Human Computer-Interaction (HCI). In order to cover all points of definition, the HCI must cover different areas including aspects of the human being and the computer. The most relevant and significant areas include computer science, industrial design, cognitive psychology and 
sociology " [2].

The HCI studies [2] hardware and software and how they affect interaction; users' mental models versus the machine model; the tasks performed by the system and its adaptation to the needs of the user; the design, which should be directed at the user and not the machine (user-centered design); and the organizational impact, which should be positive.

We can see that this term incorporates in the computer industry concepts that detach from psychology, sociology and industrial design, which have to do with a subjective aspect such as the mental models of users or the needs they have.

A few years after this term was born, the concept of engineering of usability arises from the hand of the democratization of information in the 1990s, which proposes a more strategic and economic vision of generating a good User Experience (UX). It focuses on ROI (Return On Investment), obtained results and cost-benefit ratio [3].

The concept of usability evolved over the years to become a method for assessing the quality of an interactive design, and is defined in the ISO/IEC 25010 standard [4] as The ability of the software product to be understood, learned, used and attractive to the user, when is used under certain conditions. This feature is further subdivided into the following sub-features:

- Ability to recognize its suitability. Product capability that allows the user to understand if the software is suitable for their needs.

- Learning capacity. Product capability that allows the user to learn their application.

- Ability to be used. Product capability that allows the user to operate and control it with ease.

- Protection against user errors. System capability to protect users from making mistakes.

- User interface aesthetics. User interface capability to please and satisfy user interaction.

- Accessibility. Capacity of the product that allows it to be used by users with certain characteristics and disabilities.

From another point of view, Allanwood and Beare [5] argue that, once finished or, better yet, during the design process of a software application, usability testing is a useful tool that can be used to discover what needs to be improved

From this perspective, asking ourselves what should we improve? is not the same as asking ourselves how can we improve the UX by making efficient use of the resources we have? The first question does not incorporate the experience that users will have. Thus, there are rules that define the UX and differentiate it from usability. The UX, according to Marc Hassenzahl [6], focuses on positive aspects of the user-product relationship; incorporates hedonic aspects and focuses on understanding and managing subjective aspects of product use. Thus, the hedonic/pragmatic model incorporates the concept of perception of interaction that can be:

Pragmatic. It refers to the perception capacity of the product to support the achievement of the objectives.

Hedonic. It refers to the perceived ability of the product to support the achievement of the main objectives, where is the "why" of the action that the user tries to complete.

This UX vision raises the following principle: "It is not antagonistic to distinguish between a complete description of an experience and the abstraction of a meta-experience". This differentiates the UX from usability, but it understands that UX is part of the user-centric design.

Being part of the user-centric design, we cannot avoid going through the foundations raised in this area by the World Wide Web Consortium onwards W3C, through the Web Accessibility Initiative (WAI). In 1999 published, in the Web Content Accessibility Guidelines (WCAG) document, 14 principles with the aim of promoting accessibility by focusing not only on the capabilities of each user, but also on the devices used to consume the content [7]. 
Digital Transformation: A Quality Model Based on ISO 25010 and User Experience Debnath et al.

The 2008 revision, WCAG 2.0, which was then approved as standard [8] in ISO/IEC 40500:2012, sets out 4 principles with their respective guidelines for achieving content accessibility [9]. These principles include: Perceptibility, Operability, Understanding, and Robustness. These principles have not been written for a particular technology, which makes them generalizable for all software interface developments. The WCAG2ICT guide describes how to apply them on other platforms [10]. In 2018, a new review, WCAG 2.1, was released that attempts to resolve issues of its own new available technologies [11].

Thus, if we analyze these 4 principles in depth, we can notice how the User-Centered Design is directly linked to the responsibility of the UX team to understand the user and to know their context in a certain way. The technology application modified distribution channels, transformed contact with users and accelerated processes. Thus, in addition to the management of new tools, specialists in this area must be able to interpret the context and be prepared to be protagonists of change. In this context, this paper presents a quality model of software product oriented to the UX. For the development of the proposed model, we take the following into account:

1. The structure of the International Standard ISO 13407:1999 [12], describing how a UserCentered Design process can be implemented to achieve usable systems; and its ISO 9241210:2010 review confirmed in 2015 [1, 13],

2. The ISO 25000 [14] standard, which is the cornerstone around which the system is established for the evaluation of product quality, and

3. Information collected from the Software and Computer Services Industry in the region.

\section{Quality Models}

Searching quality for software products starts from the models proposed by McCall (1976) [15], where product quality is broken down into 11 categories or quality factors by grouping them into three categories: operation, review and transition of products; Evans (1987) [16], Deustch (1988) [17], added new categories and quality factors to those already proposed. These models were developed following quality guidelines that emerged for the improvement of other types of products [18, 19]. Today, different families of quality standards oriented to software products also evolved. ISO/IEC 9126 and ISO/IEC 14598 shaped the ISO/IEC 25000 family, a family of standards also known as SQuaRE (Software Product Quality Requirements and Evaluation) [19]. This is how the 25000 family of standards is organized into five points: requirements, management, model, measurement and quality assessment, the one corresponding to the quality model -ISO/IEC 25010- is required contractually. The standard proposes three models: software product quality model, system quality of use and data quality model. The development of ontology is based on what is proposed by ISO 25010. This standard divides software product quality into 8 features (Figure 1): Functionality, Reliability, Efficiency, Usability, Security, Compatibility, Maintainability, and Portability.

For the proposed research development, we focused on Usability, the fourth feature. It establishes the ability of the software product to be understood, learned, used and attractive to the user, when used under certain conditions [4]. This feature is subdivided into: Ability to recognize your suitability, Learning ability, Ability to use, Protection against user errors, User Interface Aesthetics, and Accessibility. 
Digital Transformation: A Quality Model Based on ISO 25010 and User Experience Debnath et al.

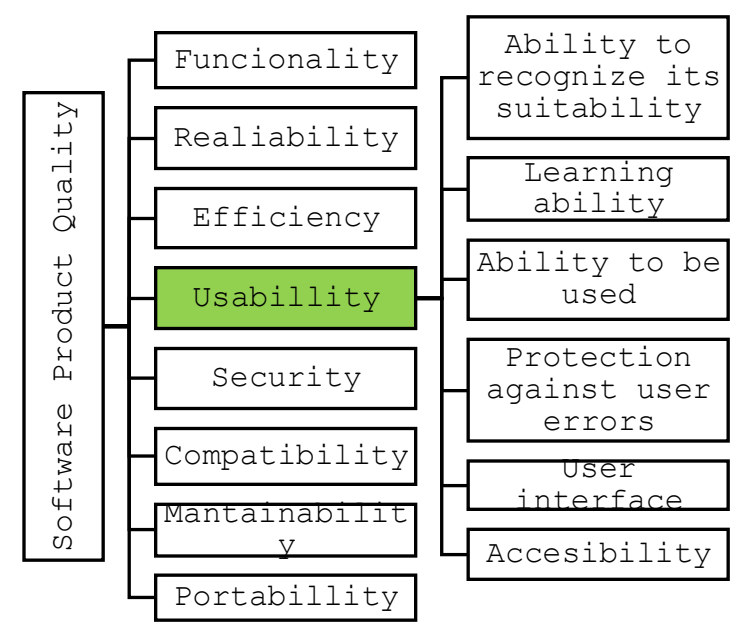

Figure 1: Software Product Quality Model.

\section{Business Models}

Recently, B2B (Business to Business), B2C (Business to consumer), B2B2C (Business to Business to consumer) business models are migrating to a D2C in which the contact is 'Direct to Consumer'. So, they have to integrate the entire value chain and the proposal to speak to the person who buys their products, even if they do not sell them directly. The scenario has been modified. Developing a digital product without asking to who will use it, for what, from which devices, etc., only moves away from the premise that reigns today in this digital age: all by the user.

Often in the software industry we find digital products that are devised and developed by people who are experts in the subject, who are easy to understand the use of a burger menu or who live in everyday life with terms such as scroll, input or form. But what if we talk about users who are not experts in the subject? What if we talk about the different age groups? about vulnerable or unwellaccessed communities to technology? How do you feel when you come across a digital product that was not devised or developed for your situation or context?

At this point, it is reasonable to analyze: how could development teams bring a digital product into the market with the user in mind if they usually work against the clock? If you often know the user in the course of development, could you cover the modifications that are being requested? To the responses we are encouraged that the teams are made up of people with different visions, and that they can think of different solutions according to their experience How could a consensus be reached?

And if we take into account that development teams often have to produce cross-platform software, impacting directly on the UX by the risks of the technologies that are used [20], could we come up with a digital product with which everyone, equipment and users, feel satisfied and identified?

Reflecting on the responses that emerged, the following conclusion was obtained: to carry out the steps of the proposed activity, but backwards would have facilitated the development and implementation of the menu, optimizing the use of resources (human, economic, material, etc.) and achieving a memorable UX. Based on this reflection, the following steps can be proposed for the process of designing a user-centric solution.

1. Analyze the problem and take the time to ask us in what context the product will be used and meet those who will use it. Raising questions to consider that allow us to propose ideas. 
2. To know the context in depth, to ask ourselves which of the proposals we thought and devised will be the most appropriate and optimal.

3. Understand the user and its features. Put us in his shoes. Make tests with the option selected for development, and thus check if the decision was the right one.

4. Knowing the context and potential users, and having carried out a preliminary test, this will be an instance to polish the proposals that emerged throughout the process. Being clear that we will achieve an excellent UX, thus improving the quality of our digital product.

To achieve this, we are working on the definition of models, methods, guides, and strategies that allow evaluating the quality of interfaces with a focus on UXs.

\section{$4 \quad$ Proposed Quality Model}

When carrying out any type of software development, knowing the context and the users that will use our digital product will give us the possibility of defining our design and development field, optimizing efforts and resources, to obtain a quality product, ensuring improve the user-product relationship. In this sense, at the end of the development of a digital product, we might ask ourselves a few questions as Does it give the user something? Is it noticeable, operable and compressible? Does the user have a positive experience? Did we achieve a robust final product? If the answer to all those questions is "yes", we will be sure to be facing a memorable UX. That is, in other words, to begin to understand that, if we talk about quality in the area of software, we talk about UX.

The quality of a software product from the customer's point of view is defined as "the degree to which a customer and/or user perceives that the software product meets their needs" [1]. Under this concept, quality standards are established. In 2005 the IOS/IEC 25000 [14] standard emerged to measure the quality of the software itself; and while the standard allows you to monitor and evaluate the quality of a product, the costs of its certification and implementation are high for small software companies.

This work introduces a quality model that bases its structure and design on the usability characteristic of the ISO/IEC 25000 standards. The model covers UX and satisfies the need to adapt to new business paradigms, considering technological and market changes. In short, offer an instrument that allows you to know the domain with which you work, get acquainted with the vocabulary of the organization and establish the tools, platforms, techniques and communication methods necessary to achieve a software that meets the customer's needs. The following is the proposed Quality Model along with a brief description:

1. Knowledge of your customers

1.1. Online Behavior

1.2. Particular Interests

2. Using Tools

2.1. Efficiency and Performance

2.1.1. Data Analysis

2.2. Social Communication Channels

2.2.1. External Platforms

2.2.1.1. Social Media

2.2.1.2. Network files tools management

2.2.2. Internal Platforms 
Below is a brief definition of each of the characteristics of the proposed model:

1. Consumers are now more informed than ever and share their own product experiences and recommendations, becoming opinion leaders. Marketing specialists should be present in the channels where they make their purchasing decisions by partnering with influencers to gain their attention and take them to the desired goal.

1.1. Omnichannel solutions that allow connecting all the processes that support each consumer contact point.

1.2. Not all users are looking for the same, nor are they reported in the same way. When the company understands that, according to the journey, there are different consumer profiles at different stages where they interact with the firm or make decisions, it is important how it does to understand what they need.

2. Algorithms that study the user to impact him with recommendations and related products in the phases of brand knowledge and consideration, which increase conversions on digital platforms.

2.1.1. A key technique is data driven, i.e. data analysis that allows decisions to be made with Customer Centric in mind.

2.2. Communication Channels: From this scenario, it is very convenient that from now on, you try to detect the tools that best defend the interests of your company or digital activity.

2.2.1. External Platforms: Suites or software that can be used through a monthly subscription. They have as advantages that they offer a service of help and attention to the client.

2.2.1.1. Social networks. Certain instruments that allows programming your posts or other materials in the information.

2.2.1.2. Tools for files on the network, such as Google Drive or Dropbox.

2.2.2. Internal Platforms: The organization's own development software. It has the characteristic that they are deployed on servers in the cloud or on their own servers, with the necessary functionalities for what corresponds to digital marketing.

Use of tools connects the consumer experience with business outcomes. In summary, after research and consultation with marketing professionals from different places in Argentina, it was concluded that it is necessary to have a set of minimum basic tools to be able to position the company and adapt to the times today, having the customer as the center of commercial interest. Some of these tools include:

Social Networks Management, Suite Tools of Marketing, Sales and Customer Support, To Find Viral Content and Influential Persons, Tell Highlighting Stories, Mail Marketing Service Provider, a web analytics tool.

The quality model is accompanied by a set of metrics and indicators that provide the intelligence organization to provide greater decision-making efficiency. Some examples are shown below.

NTSN: Number of Tools that Manage Social Networks in your organization.

NSMS\&S: Number of Suite of marketing, sales and customer service tools.

NTVC: Number of tools that allows finding viral content.

NTSI: Number of tools to search for influencers.

NTHS: Numbers of tools to tell highlight stories.

NeMSP: Number of email marketing service providers.

NWAT: Number of web analytics tools.

From these direct metrics, the derived metric Percentage of Tool Uses (PTU) is defined as: 
Digital Transformation: A Quality Model Based on ISO 25010 and User Experience Debnath et al.

$($ PTU $)=(($ NTSN + NSMS\&S + NTVC + NTSI + NTHS + NeMSP + NWAT $) /$ Number of Total Tools

To interpret the PTU metric, the elementary indicator is defined:

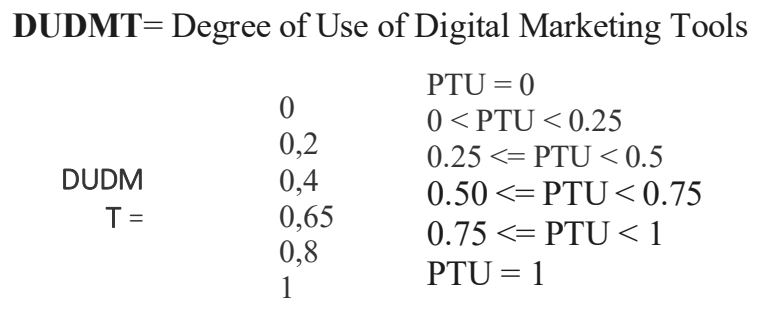

\section{Case Study on Digital Marketing Process: Migration to the Cloud and Its Business Processes}

A decision was taken to restructure the business processes adapting them to the new technological demands. In this context, as a first step, a cloud internet provider has been hired. But that had an adaptation problem for the company's work teams. The migration was challenged because different requirements have to be achieved by the company's software and, in addition, adjust the validation and verification processes to meet quality assurance. It was also necessary to control the external part: the different applications, repositories, etc. that emerged in the new paradigm of the cloud. To this end, the company's human resource was reorganized with the use of a strategy. To complete the strategy, we had to think about how to design or condition the interfaces of web and mobile applications in order to adapt to the new business paradigms. That is, maintain a leading position in the market.

For this work, the quality model previously defined was used with efficiency and performance tools in mind. Focused on the data analysis, which is one of the sub-features of interest when it comes to the customer. To this end, one of the first activities carried out was the analysis of the data/information that was held of customer preferences. A study was made of the documents that are used to record the results of the business processes. To do this, the amount of advertising targeted on time to each customer was counted, based on their preferences or needs. From purchases, budget orders, traceability was made with their records and the advertisements, personalized recommendations that were made. The result was practically nil. It could be found that employees could have an approximation of customer behavior. But there was a marked lack of digital information in terms of it. There were few features or queries about it. Table 1 shows a summary of the measures you take out.

\begin{tabular}{|c|c|c|}
\hline Customer Knowledge & Scope & Percentage \\
\hline Behavior & Physical & $62,5 \%$ \\
\hline & Online & $5 \%$ \\
\hline Particular Interests & Preferences & $57 \%$ \\
\hline & Targeted Advertisement & $0 \%$ \\
\hline
\end{tabular}

Table1: Measures Summary

In Table 1, we worked with the characteristics and sub-features of the quality model such as: Behavior and Particular Interests. With regards to the behavior, online and physical or face-to-face behavior in the company was measured through the metrics defined for this purpose. A percentage of 
the customer's fingerprints were searched in the database in terms of the organization's online presence. It was very scarce, but at this point it was possible to notice that the company's website was intended for another time, this was made visible by the little information that was recorded of the customer's browsing. Measurements and observations using different instruments could find that physical behavior, which was assimilated by the knowledge of sellers, was more specific and of higher quality than online. Therefore, advertising addressed to the interests or tastes of the customer only occurred in the sales room through the sellers/employees, who could record in their memory the preferences of each customer. This highlighted the need to carry out more personalized work through different instruments, methods or techniques that will bring us closer to customers.

Based on this, the organization should strive to understand how to adapt its sales strategies to the consumption habits of the "Millennial" generation. Much is said about these digital natives: they are more critical and demanding, they prioritize their shopping experience, they buy more from their smartphones and that they have a lot of dedication and smell when looking for promotions and discounts.

But how much does this behavior differ from other consumers? A recent study by Deloitte [21] indicates differences in consumption habits between millennials and non-millennials are increasingly declining. Despite not being digital natives, the latter adapted and adopted consumption patterns typical of the younger ones. In fact, the study indicates that $52 \%$ of non-millennials incorporated electronic stores as part of their shopping habits and grew by 8 points, almost matching the rate of use of millennials.

Faced with this, organizations that have a good omnichannel strategy based on their customers' knowledge and the customization of the offer will be able to bring these end consumers product offerings that are relevant to them. Knowing the customer and their behavior, especially in e-commerce, is an essential task for organizations/companies to generate the best possible shopping experience, while increasing their sales.

To make sales strategies more effective, the major commitment of organizations is on automated customization, learning from every customer interaction. In e-commerce, the time of searching for a product is critical, because if you do not get adequate results the customer ends up leaving the site. Intelligent customization allows the customer to find relevant products faster, and also that when bidding through automated personalized email marketing, they receive product information relevant to their interests.

According to a report conducted by the Data \& Marketing Association in the United Kingdom [22], $58 \%$ of the revenue of the companies surveyed came from personalized emails according to segments and prior interests of users. Effectiveness increases when customization is not by audiences and by adherence to consumer behavior on the site. Specifically, the promotion of the products in our case was only done through the website and an email. With which, it was intended to approach the client in a more personalized way. This meant that we had to search for messages sent and draw conclusions. This meant that there were no specific tools for data/information/knowledge analysis. Therefore, only $15 \%$ of these tools or instruments were registered, as regards the characteristic performance tools of our quality model with regards to data analysis. Communication channels are another important feature of marketing, reflected in the proposed quality model. This feature is subdivided into sub-features such as External and Internal Platforms or Organization-specific Platforms. As external platforms, a social network was used to some extent and very informally and dependent on an employee. It was also found that, at the time of the study, no regular network tools were used for information management. The strong measurement and study lay on the subject of institutional email as an internal platform of the organization. But the analysis of data and information was done by hand. Table 2 shows a summary of the results obtained.

In this sense, as a recommendation or suggestion to the company it was reported that the tools allow organizations to adapt their display cases according to the browsing behavior of each client to offer them information and products in a way according to the customer's behavior on the site. 


\begin{tabular}{|c|c|c|c|}
\hline Type of Tools & Use & Type of Tools & Percentage \\
\hline Efficiency and Performance & Data Analysis & & $15 \%$ \\
\hline Communication Channels & External Platforms & Social Networks & $7 \%$ \\
\hline & & Networks Tools & $5 \%$ \\
\hline & Internal Platforms & & $30 \%$ \\
\hline
\end{tabular}

Table 2: Analysis Results

In turn, it gives companies the ability to bring personalized recommendations to email marketing campaigns with content and promotions focused on consumer interests automatically. The goal: to increase conversion rates, profitability and not to exhaust potential consumers with irrelevant information.

Taking advantage of the opportunities that organizations/companies have to reach their customers with personalized and relevant offers will be a key when capitalizing on the user's browsing moment. In this way, they will be able to discover the products that they want to buy more easily, that their experience in the display case leads them to make the purchase decision and that it can then be complemented with cross-selling. For this, organizations/companies must first know their customers, their online behavior and their particular interests.

\section{Conclusions}

Nowadays, a good communication strategy is increasingly needed consisting of something very important: if people do not know the new products are offered in their points of sale, or the benefits they offer when subscribing to their newsletter, or follow your social networks, breaking this online/offline barrier will become increasingly difficult.

There is much talk about the concept of "Infinity channel", a strategy that makes commercial brands put in the new generations shoes that focus on living all omnichannel experiences alike. Due to this scenario, it is becoming increasingly clear that the barriers between the physical and online world must be set aside.

However, not all users are looking for the same, nor are they reported in the same way. When the company understands that, according to the journey, there are different profiles of consumers at different stages where they interact with the firm or make decisions, it is important what is done to understand what its users need.

From this perspective, it helps to be able to measure and evaluate the quality of processes and software products from the UX point of view. The current research work proposed a quality model based on existing quality standards and oriented to the UX. This model was instantiated to evaluate the quality of a company's processes and during the migration of its business processes to the cloud.

Thus, after instantiating the quality model and applying the metrics and indicators; it was concluded that, in the project of migrating to the cloud and transforming the organization to a digital organization, major adjustments and transformations were needed in terms of business processes. It was necessary to hire HootSuite which is a web and mobile platform to manage social networks by people or organizations. HootSuite allows one to use several social networks such as Facebook, Twitter, LinkedIn, Google, Instagram, YouTube, and Foursquare. In this way is possible to reach the client from various sources and ways it was decided to start with this application for the management of the two or three instruments that would start to be used in the organization.

It also turned out that the data and information were there, but first the structure of the database had to be conditioned, and then start to work with some platform or software for the analysis of the data that would be collected from customers. In this first instance we opted for the selection of Google Analytics which is a web analytics tool of the company Google. This tool provides pooled information about the 
traffic that reaches the websites based on the audience, acquisition, behavior, and conversions that take place on the website.

As future works, it remains pending the development of an application by the development team that allows employees, sellers, salespeople and other members to centralize in it all the knowledge of each customer. It is necessary to make those changes and think of some strategy that allows to adapt to the new business paradigms, today named as 4.0 .

\section{References}

[1] ISO 9241-210:2010 Ergonomics of human-system interaction -- Part 210: Human-centred design for interactive systems, 2010.

[2] M. C. Marcos, "HCI (human computer interaction): concepto y desarrollo," in El Profesional de la Información, vol. 10, 2001.

[3] J. Nielsen, "Usability Engineering," Mountain View, California, , 1993.

[4] ISO/IEC 25010:2011 Systems and software engineering -- Systems and software Quality Requirements and Evaluation - System and software quality models, 2011.

[5] G. Allanwood, P. Beare; Diseño de Experiencias de Usuario. 2015, pp. 12-36.

[6] M. Hassenzahl; "The Hedonic/Pragmatic Model of User Experience," Landau, 2007.

[7] W. W. W. Consortium, "Web Content Accessibility Guidelines 1.0". [On line]. Available: http://www.w3.org/TR/1999/WAI-WEBCONTENT-19990505. [Last access: March, 2019]. 1999.

[8] I. O. f. S. I. E. Commission; "ISO/IEC 40500:2012 Information technology -- W3C Web Content Accessibility Guidelines (WCAG) 2.0," 2012.

[9] W. W. W. Consortium; "Web Content Accessibility Guidelines (WCAG) 2.0.". [On line]. Available: http://www.w3.org/TR/2008/REC-WCAG20-20081211/. [Last access: March, 2019]. 2008 .

[10] W. W. W. Consortium; "Guidance on Applying WCAG 2.0 to Non-Web Information and Communications Technologies (WCAG2ICT)". [On line]. Available: http://www.w3.org/TR/2013/NOTE-wcag2ict-20130905/. [Last access: March, 2019]. 2013.

[11] W. W. W. Consortium; "Web Content Accessibility Guidelines (WCAG) 2.1". [On line]. Available: https://www.w3.org/TR/2018/REC-WCAG21-20180605/. [Last access: March, 2019]. 2018.

[12] ISO; ISO 13407:1999, 1999.

[13] ISO; ISO 9241-11:2018, 2018.

[14] ISO; ISO/IEC 25000. SQuaRE - System and Software Quality Requirements and Evaluation.

[15] J. McCall, P. Richards; "Factors in software quality. US Rome Air Development Center Reports", 1977. 
Digital Transformation: A Quality Model Based on ISO 25010 and User Experience Debnath et al.

[16] M. Evans, J. Marciniak; "Software Quality Assurance and Management". Jhon Willey \& Sons. 1987.

[17] M. S. Deustch, R. R. Willis; "Software Quality Engineering, A Total Technical Management Approach. Prentice Hall.," 1988.

[18] M. Sangüesa, R. Mateo, L. Hzarbe; "Teoría y Práctica de la Calidad". España: Thomson., 2006.

[19] M. G. Piattini Velthuis, F. O. García Rubio, I. García Rodriguez de Guzmán, F. Pino; Calidad de Sistemas de Información. 2012.

[20] A. Biørn-Hansen, T.-M. Grønli, G. Ghinea, S. Alouneh; "An Empirical Study of CrossPlatform Mobile," Wireless Communications and Mobile Computing, vol. 2019, $\mathrm{n}^{\mathrm{o}}$ 5743892, 3, 2019.

[21] Deloitte; "Big demands and high expectations The Deloitte Millennial Survey," Executive summary. On Line. [Last access september, 2019], 2014.

[22] (2019). Data \& Marketing Association. Available: https://thedma.org. [Last access september, 2019]. 